\title{
Observation of Skyrmions at Room-temperature in Amorphous Fe/Gd Films
}

Jordan Chess $^{1}$, Sergio Montoya ${ }^{2}$, James Lee ${ }^{1,3}$, Sujoy Roy ${ }^{3}$, Steven Kevan ${ }^{1,3}$, Eric Fullerton ${ }^{2}$, Ben McMorran $^{1}$

1. Department of Physics, University of Oregon, Eugene

2. Center for Magnetic Recording Research, University of California, San Diego

3. Lawrence Berkeley National Laboratory

We have employed Lorentz TEM (LTEM) and focal series reconstruction at multiple sample tilt angles and applied fields to investigate magnetic structures in amorphous Fe/Gd thin films.

Magnetic bubbles, investigated in the 1970 s as potential data storage media ${ }^{1}$, have seen a recent surge in scientific inquiry due to the their topological spin textures [1]. A specific type of bubble with whirling magnetic spins (see Fig. 1) called a skyrmion is the major driving force behind these investigations. Skyrmions are characterized by a non-zero chirality or topological charge, given by $N_{s}=\int \mathbf{m}$. $\left(\partial_{x} \mathbf{m} \times \partial_{y} \mathbf{m}\right) d x d y$, where $\mathbf{m}$ is the normalized in-plane magnetization $\mathbf{m}=\mathbf{M}(x, y) /|\mathbf{M}(x, y)|$. These spin textures were first observed in MnSi using neutron scattering [2-4]. Much of the excitement surrounding skyrmions is due to their high potential for application in spintronics [5,6]. Yu et al. observed skyrmion motion in FeGe using LTEM at current densities as low as $10 \mathrm{~A} \cdot \mathrm{m}^{-2}, \sim 10^{6}$ orders of magnitude lower than that required to move domain walls in ferromagnetic devices making them promising for racetrack memory applications [5-7]. While previous magnetic skyrmion research has largely been focused on non-centrosymmetric crystals where the Dzyaloshinskii-Moriya interaction (DMI) stabilizes the skyrmion phase, here we present a LTEM study of an amorphous ferromagnetic material with perpendicular magnetic anisotropy, consisting of an Fe/Gd multilayer film.

$\mathrm{Fe}_{\mathrm{a}} \mathrm{Gd}_{\mathrm{b}}$ films with $\mathrm{a}=3.4,3.5,3.6 \AA \mathrm{b}=4.0 \AA$ and 80 repeating layers were produced by sputter deposition onto a SiN window. Data was recorded in Lorentz mode using an FEI Titan equipped with an image aberration corrector. By preforming transport-of-intensity equation (TIE) analysis, the under- and over-focus LTEM images can be used to determine the in-plane magnetic induction of the sample. At zero applied field all samples have magnetic stripe/labyrinth domains shown in Figures 1b and 1c. Increasing the magnetic field applied out-of-plane causes the stripe or labyrinth domains to break up into magnetic bubble structures shown in Figure 2. These structures can be placed in three categories: type II magnetic bubbles, skyrmions, and biskyrmions, Figure 2 has examples of these three structures. Biskyrmions are a composite state formed by two skyrmions with opposite chirality for which the total topological charge is 2, and they have only been observed in $\mathrm{La}_{2-2 x} \mathrm{Sr}_{1+2 x} \mathrm{Mn}_{2} \mathrm{O}_{7}$ by $\mathrm{Yu}$ et al. [8]. As seen in Figures $2 \mathrm{c}$ and $\mathrm{j}$ the type II bubbles and biskyrmions have very similar spin textures. Additionally, both these structures form in lines where stripe domains previously resided, similar to what was observed in $\mathrm{La}_{2-2 x} \mathrm{Sr}_{1+2 x} \mathrm{Mn}_{2} \mathrm{O}_{7}$ [8]. The presence of biskyrmions and skyrmions with both helicities indicates that in this material these topological spin textures are stabilized by long range dipole interactions, as opposed to the Dzyaloshinskii-Moriya interaction responsible for stabilizing the skyrmion phase in non-centrosymmetric crystals such as MnSi and FeGe [9].

To further investigate the stability of these magnetic structures we have varied both the magnetic field strength and tilt angle of the samples. We found that the type of magnetic feature (stripe, labyrinth, skyrmions...) that appears is largely determined by the detailed field history of the sample. For example, 
tilting the sample to $30^{\circ}$, applying a saturating field, then reducing the field back to zero produces a nearly uniform stripe domain similar to that seen in Figure 1b. We also observe that uniform stripe domain patterns favor the creation of biskyrmions and type II bubbles when an out-of-plane field is applied, while the skyrmion state is favored when the film starts in a labyrinth pattern. These results give further evidence that these magnetic structures are stabilized by long range magnetic dipole interactions.
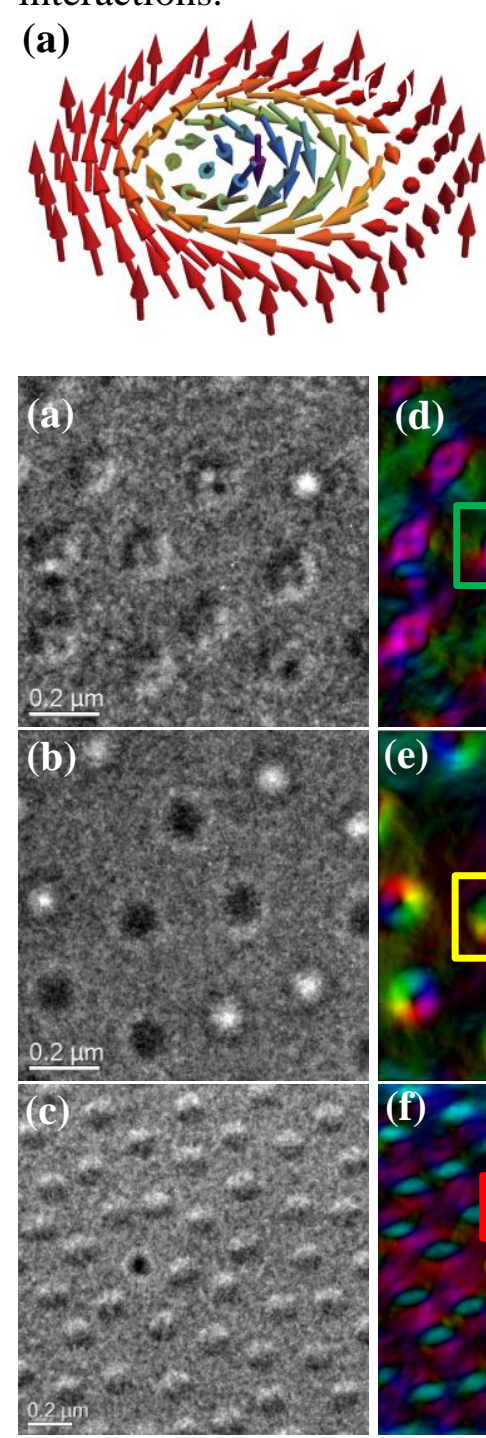
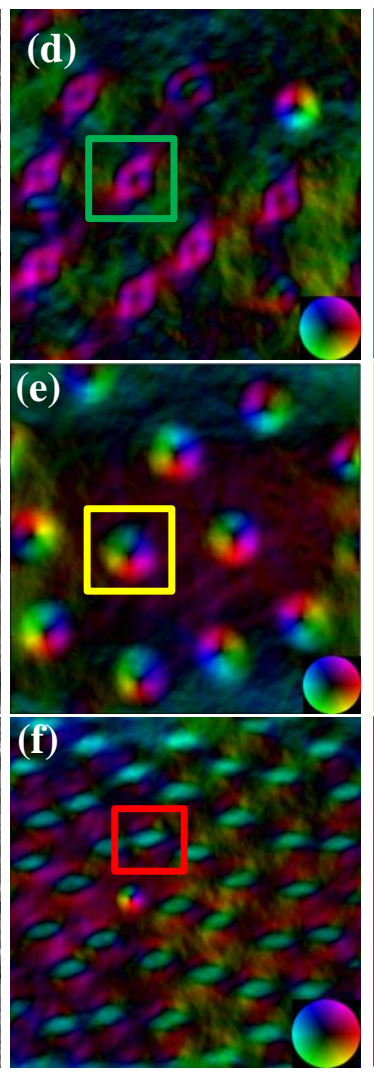
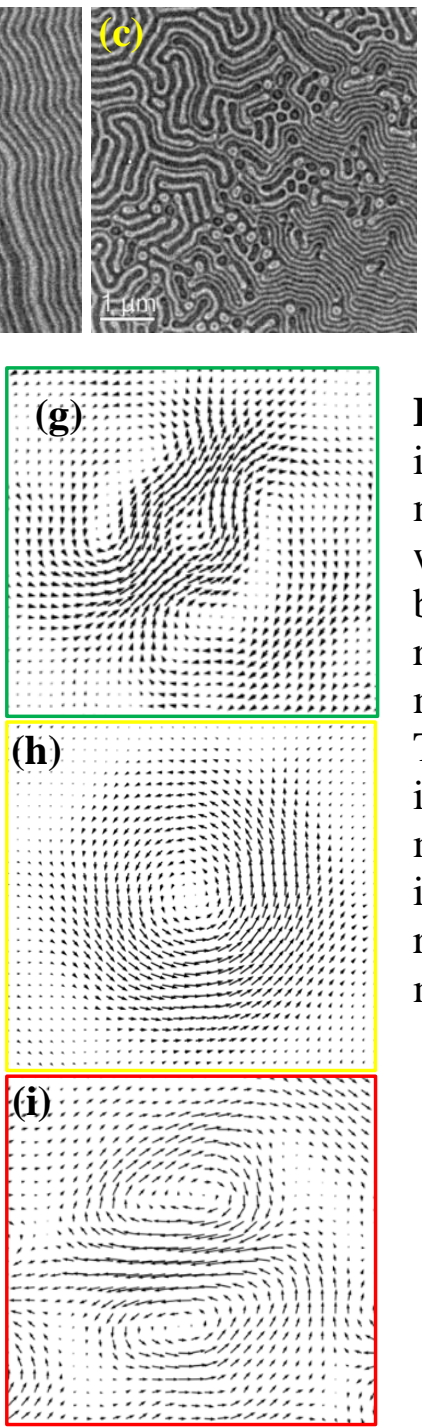

Figure 1. (a) Spin structure of a Bloch type skyrmion, underfocus Fresnel contrast Lorentz microscopy images showing (b) stripe domain pattern, (c) labyrinth domain pattern

Figure 2. (a-c) under-focus Lorentz images showing (a) type II magnetic bubbles, (b) skyrmions with both helicities and (c) biskyrmions. (d-f) color representations of the in-plane magnetic induction calculated using TIE analysis hue and saturation indicate the direction and magnitude of the magnetic induction. (g-i) vectorial representations of the in-plane magnetic induction.

References:

[1] Malozemoff, A. \& Slonczewski, J. "Magnetic Domain Walls in Bubble Materials: Advances in Materials and Device Research", ed. R Wolfe (Academic press, New York).

[2] Mühlbauer, S. et al. Science 323, 915-919 (2009).

[3] Neubauer, A. et al. Phys. Rev. Lett. 102, 186602 (2009).

[4] Pappas, C. et al. Phys. Rev. Lett. 102, 197202 (2009).

[5] Fert, A., Cros, V. \& Sampaio, J. Nat. Nanotechnol. 8, 152-156 (2013).

[6] Tomasello, R. et al. Sci. Rep. 4, (2014).

[7] Yu, X. Z. et al. Nat. Commun. 3, 988 (2012).

[8] Yu, X. Z. et al. Nat. Commun. 5, (2014).

[9] Yu, X. et al. Proc. Natl. Acad. Sci. U. S. A. 109, 8856-8860 (2012). 